\title{
Vagotomy Affects the Development of Oral Tolerance and Increases Susceptibility to Develop Colitis Independently of $\alpha-7$ Nicotinic Receptor
}

\author{
Martina Di Giovangiulio, Goele Bosmans, Elisa Meroni, Nathalie Stakenborg, Morgane Florens, \\ Giovanna Farro, Pedro J Gomez-Pinilla, Gianluca Matteoli, and Guy E Boeckxstaens
}

Department of Clinical and Experimental Medicine, Translational Research Center for Gastrointestinal Disorders (TARGID), Division of Gastroenterology, KU Leuven, Leuven, Belgium

\begin{abstract}
Vagotomy (VGX) increases the susceptibility to develop colitis suggesting a crucial role for the cholinergic anti-inflammatory pathway in the regulation of the immune responses. Since oral tolerance and the generation of regulatory $T$ cells (Tregs) are crucial to preserve mucosal immune homeostasis, we studied the effect of vagotomy and the involvement of $\alpha 7$ nicotinic receptors ( $\alpha 7 n A C h R)$ at the steady state and during colitis. Therefore, the development of both oral tolerance and colitis (induced by dextran sulfate sodium (DSS) or via T cell transfer) was studied in vagotomized mice and in $\alpha 7 n A C h R^{-1-}$ mice. VGX, but not $\alpha 7 n A C h R$ deficiency, prevented oral tolerance establishment. This effect was associated with reduced Treg conversion in the lamina propria and mesenteric lymphnodes. To the same extent, vagotomized mice, but not $\alpha 7 \mathrm{nAChR^{-/ }}$ mice, developed a more severe DSS colitis compared with control mice treated with DSS, associated with a decreased number of colonic Tregs. However, neither VGX nor absence of $\alpha 7 n A C h R$ in recipient mice affected colitis development in the T cell transfer model. In line, deficiency of $\alpha 7 n A C h R$ exclusively in T cells did not influence the development of colitis induced by T cell transfer. Our results indicate a key role for the vagal intestinal innervation in the development of oral tolerance and colitis, most likely by modulating induction of Tregs independently of $\alpha 7 n A C h R$.
\end{abstract}

Online address: http://www.molmed.org

doi: $10.2119 / \mathrm{molmed} .2016 .00062$

\section{INTRODUCTION}

Recent studies have now undoubtedly demonstrated that the nervous system extensively interacts with the immune system to modulate systemic and peripheral inflammation (1). In 2000, Tracey and co-workers elegantly showed that vagus nerve stimulation (VNS) reduced proinflammatory cytokine release and increased survival in a model of sepsis (2). This antiinflammatory effect, called cholinergic antiinflammatory pathway (CAIP), resulted in inhibition of splenic macrophages (M $\varphi s$ ) through the activation of alpha7 nicotinic acetylcholine receptors $(\alpha 7 \mathrm{nAChR})(3,4)$. In 2005, we extended this concept to the gastrointestinal (GI) tract showing that VNS reduced inflammation and restored GI transit in a murine model of postoperative ileus (POI) (5). In the context of POI, we recently demonstrated that VNS

${ }^{*} G E B$ and GM should be considered as co-last authors.

Address correspondence to Guy E Boeckxstaens or Gianluca Matteoli, Department of Clinical and Experimental Medicine Translational Research Center for Gastrointestinal Disorders (TARGID) KU Leuven, Herestraat 49, O\&N1 bus 701, 3000 Leuven, Belgium. Tel:+32-16-377566; E-mail: Guy.Boeckxstaens@med.kuleuven.be or Gianluca.matteoli@ med.kuleuven.be.

Submitted March 11, 2016; Accepted for Publication May 31, 2016; Published Online (www.molmed.org) June 14, 2016.

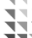

Feinstein Institute

for Medical Research

Northwell Health

exerts its antiinflammatory effect in the intestinal muscularis externa by activating cholinergic enteric neurons in close contact with CX3CR1 ${ }^{\text {high }} \alpha 7 \mathrm{nAChR}{ }^{+}$ resident $\mathrm{M} \varphi \mathrm{s}$ (6). Thus, cholinergic innervation has a major impact on the immune system in the intestinal muscularis. Moreover, vagotomy (VGX) has been reported to increase the basal expression of NF-kB and the production of proinflammatory cytokines in the lamina propria and to increase the susceptibility to develop colitis in murine models (7-13). In fact, macroscopic and microscopic parameters such as histologic scores, myeloperoxidase activity and levels of proinflammatory cytokines in colon were increased in vagotomized mice during chemically and hapten-induced colitis (8). However, the exact mechanism underlying this increased susceptibility to develop colitis is still unclear. In the present study, we hypothesized that increased susceptibility to develop colitis following 
VGX may result from alteration of regulatory $\mathrm{T}$ cells (Tregs) population.

Tregs are involved in the control of mucosal immune responses preventing the development of chronic intestinal inflammation as in colitis. Tregs are abundantly distributed in the lamina propria of the small intestine and colon, in which they control the immune response against dietary and microbial antigens. Tregs significantly contribute to mucosal immune homeostasis through the installation of oral tolerance, a phenomenon in which local and systemic immune unresponsiveness is induced by oral administration of an innocuous antigen (14). Antigen-specific Tregs are generated by sequence of events starting with antigen uptake by tolerogenic $\mathrm{CD}_{103^{+}}$dendritic cells (DCs) in the lamina propria. At the steady state $\mathrm{CD}_{103}{ }^{+}$DCs migrate to mesenteric lymph nodes (MLNs) where naïve $\mathrm{T}$ cells are converted into CD4 ${ }^{+}$ $\mathrm{CD}_{25}{ }^{+} \mathrm{FoxP}^{+}$Tregs. These antigen-specific Tregs successively migrate back to the lamina propria in which they are expanded by CX3CR $1^{\text {high }} \mathrm{M} \varphi$ s in interleukin (IL)-10-dependent fashion (15). Interference with antigen-specific Tregs conversion in the gut increases susceptibility to develop chronic intestinal inflammation. Indeed, adoptive transfer of naïve Th cells (deprived of the Treg counterpart) into immune deficient mice typically results into the development of colitis (16). In line, in a model of spontaneous colitis the severity of the disease was associated to altered intestinal Tregs development (17). Taken together, these data indicate that beside their importance in installing oral tolerance, Tregs determine the susceptibility to develop colitis.

In the present study, we investigated the effects of VGX on Tregs population and on the susceptibility to develop colitis. Since $\alpha 7 \mathrm{nAChR}$ has been shown to mediate the effects of the CAIP activation on CX3CR $1^{\text {high }} \mathrm{M} \varphi \mathrm{s}$ (18), we also evaluated the involvement of this receptor using $\alpha 7 \mathrm{nAChR}$ knock-out $\left(\alpha 7 \mathrm{nAChR}^{-/-}\right)$mice in the development of both oral tolerance and colitis.

\section{MATERIALS AND METHODS}

\section{Animals}

$\alpha 7$ nicotinic receptor knockout $\left(\alpha 7 \mathrm{nAChR}^{-/-}\right.$; B6.129S7-Chrna $\left.{ }^{7 \mathrm{tm} 1 \mathrm{Bay}}\right)$, Rag-1 knockout (Rag1/-'; B6.129S7Rag1 $1^{\text {tm1Mom } / J) ~(19), ~ O T-I I ~ L y 5.1 ~(O T I I ; ~ L y ~}$ 5.1), Rag1 $\alpha 7 \mathrm{nAChR}$ double knockout (Rag1(KO) $\times$ Chrna7 < tm1Bay/J) and $\mathrm{CX}_{3} \mathrm{CR}_{1}^{\mathrm{GFP} / \mathrm{WT}}\left(\mathrm{C} \times 3 \mathrm{Cr}^{\text {tm1Litt }}\right)$ mice were bred at the KU Leuven animal facility in a pathogen-free environment maintained at $22^{\circ} \mathrm{C}$ in $\approx 50 \%$ relative humidity and with a 12-h light/dark cycle, while wild-type mice (WT, C57BL/6JOlaHsd) were purchased from Harlan. Mice were housed with ad libitum access to standard rodent food and water and were genotyped by PCR on total genomic DNA from the tail. All experimental procedures were approved by the Animal Care and Animal Experiments Committee of the KU Leuven (Leuven, Belgium).

\section{Experimental Protocols}

Vagotomy procedure. Mice were anesthetized by intraperitoneal (i.p.) injection of a mixture of Ketamine (Ketalar $100 \mathrm{mg} / \mathrm{kg}$; Pfizer) and Xylazine (Rompun $10 \mathrm{mg} / \mathrm{kg}$; Bayer). The skin and abdominal wall were incised along the ventral midline, and the intestines were moved aside to allow access to the left lateral lobe of the liver and the stomach. The left lateral lobe of the liver was gently retracted and stomach pulled down beneath the diaphragm to clearly expose both vagal trunks, which were then transected. All connective tissue surrounding the esophagus was removed to ensure transection of all vagal branches. The surgical pyloroplasty (PP) was used to avoid gastric dilatation due to vagotomy. As control group, mice were subjected to PP alone and vagal trunks exposed but not transected. The complete resection of both the ventral and dorsal truncal branches of the subdiaphragmatic vagus was assessed at the end of each experiment in every animal included in the study using a dissection microscope.
Oral tolerance. Mice received 3 intragastric administrations of Dulbecco's Phosphate-Buffered Saline (DPBS) as control or were sensitized with $20 \mathrm{mg}$ OVA in $200 \mu \mathrm{L}$ DPBS at d 0, 3 and 6. On d 14, all mice were immunized via subcutaneous (s.c.) injection with $50 \mu \mathrm{g}$ OVA in $100 \mu \mathrm{L}$ PBS-Complete Freud Adjuvant (CFA, Sigma-Aldrich) emulsion. After $7 \mathrm{~d}$, mice were challenged by s.c. injection of $250 \mu \mathrm{g}$ heat-aggregated OVA in $20 \mu \mathrm{L}$ of PBS in the hind footpad. OVA-specific delayed-type hypersensitivity (DTH) was determined by footpad swelling $48 \mathrm{~h}$ after challenge. Footpad swelling was measured in a blinded fashion prior to and $48 \mathrm{~h}$ after injection with an electronic digital micrometer. OVA-specific footpad swelling was calculated as footpad thickness $48 \mathrm{~h}$ footpad thickness $0 \mathrm{~h}$ and used as readout for immune tolerance to OVA.

$\mathrm{T}$ regulatory cell conversion assay. To study antigen-specific $\mathrm{T}$ regulatory cells conversion in vivo, $\alpha 7 \mathrm{nAChR}^{+/+}$, $\alpha 7 \mathrm{nAChR}^{-/-}$, vagotomy (VGX) and PP mice were adoptively transferred with $3.5 \times 10^{6}$ naïve OVA-specific CD $4^{+} \mathrm{CD} 45.1^{+} \mathrm{T}$ cells isolated from the spleen of OTII Ly5.1 mice. Briefly, $\mathrm{CD}^{+}{ }^{+} \mathrm{CD} 25^{-} \mathrm{T}$ cells from OTII Ly5.1 mice were isolated and enriched from splenocytes using magnetic beads (MiltenyiBiotec). The enriched cells were adoptively transferred into syngeneic recipient mice according to the experiment. The first and second day after injection, mice were fed with $50 \mathrm{mg}$ of OVA (grade III; Sigma-Aldrich). Five and twelve days after injection, mice were sacrificed and small intestine lamina propria and MLNs were collected from CD $45.2^{+}$hosts mice. Flow cytometry analysis of $\mathrm{T}$ cells was performed by quantification of proliferation of OVA-specific CD $45.1^{+} \mathrm{T}$ cells and conversion in Tregs. For the FoxP3 staining, cells were initially stained for the surface markers APC-conjugated anti-CD45.1 (clone A20, BD Biosciences), PeCy7-conjugated anti-CD4 (clone: RM4-5, eBioscience) and anti-Alexa488 anti-CD25 (eBio7D4, eBioscience). Subsequently, cells were fixed and permeabilized 
with the Fix/Perm buffer (eBioscience). Permeabilized cells were stained for FoxP3 (FJK-16s; eBioscience) according to the manufacturer's instructions. Samples were acquired with FACSCanto II (BD Biosciences) and analyzed with FlowJo (Treestar).

Experimental colitis. Chemicallyinduced colitis was induced in VGX, $\mathrm{PP}, \alpha 7 \mathrm{nAChR}^{-/-}$and $\alpha 7 \mathrm{nAChR}^{+/+}$mice. Mice were treated with $2 \%$ dextran sulfate sodium (DSS, TdB Consultancy AB) in drinking water for $5 \mathrm{~d}$ followed by normal drinking water until d 14. Body weight and stool consistency score were monitored daily (20). Stool consistency score was evaluated daily for the DSS colitis model and twice per week for the T cell transfer model of chronic colitis. Scoring for stool consistency was performed as follows: 0 , normal stool; 1 , loose stool; 2, diarrhea; 3, mucus in diarrhea (20).

To induce $\mathrm{T}$ cell transfer chronic colitis, naïve $\mathrm{CD}^{+}{ }^{+} \mathrm{CD} 45 \mathrm{RB}^{\text {hi }} \mathrm{T}$ cells isolated from spleens of $\alpha 7 \mathrm{nAChR}^{+/+}$and $\alpha 7 \mathrm{nAChR}^{-/-}$and C57BL/6JOlaHsd WT mice (8- to 12-wk-old) were purified via fluorescence-activated cell sorting (FACS) as previously described (21) and injected

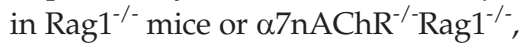
in $\alpha 7 \mathrm{nAChR}^{+/+} \mathrm{Rag}^{-/-}$mice or VGX$\mathrm{Rag}^{-/-}$and in PP-Rag1 ${ }^{-/-}$mice. In brief, after enrichment of $\mathrm{CD}^{+}$lymphocytes by magnetic beads (MiltenyiBiotec), cells were stained with PeCy7-conjugated anti-CD4, Alexa488-conjugated anti-CD25, PE-conjugated anti-CD62L and APCanti-CD45RB (all from eBioscience). Naive $\mathrm{CD}^{+} \mathrm{CD} 25^{-} \mathrm{CD} 2 \mathrm{~L}^{+} \mathrm{CD} 45 \mathrm{RB}^{\text {hi }} \mathrm{T}$ cells were purified ( $>99 \%$ ) using a FACS ARIA I (BD Biosciences). A total of $5 \times 10^{5}$ sorted naïve Th cells were injected i.p. into 10-wk-old mice.

Isolation of leukocyte subpopulations and flow cytometry analysis. Cell suspensions were prepared from the colon lamina propria, small intestine lamina propria and MLNs as previously described (22). For FoxP3 staining, cells were initially stained for the surface markers CD4 and CD25. Subsequently, cells were fixed and permeabilized with Fix/Perm buffer (eBioscience).
Permeabilized cells were stained for FoxP3 (FJK-16s; eBioscience) according to the manufacturer's instructions. The intracellular expression of IL-17A and IFN- $\gamma$ in CD4 T cells was analyzed using a Cytofix/Cytoperm Kit according to the manufacturer's instructions (BD Biosciences). In brief, lymphocytes were incubated with $50 \mathrm{ng} / \mathrm{mL}$ phorbol 12-myristate 13- acetate (PMA; SigmaAldrich), $500 \mathrm{ng} / \mathrm{mL}$ ionomycin (SigmaAldrich) and monensin (GolgiStop, BD Bioscience) in complete media at $37^{\circ} \mathrm{C}$ for $4 \mathrm{~h}$. After surface staining with CD4, cells were permeabilized and intracellular cytokine staining was performed using anti-IFN- $\gamma$ mAb (XMG1.2; BD Biosciences) and anti-IL-17A mAb (eBio17B7, eBioscience). Cells were acquired with a FACSCanto (BD Biosciences) and analyzed with FlowJo software (Treestar). To analyze antigen presenting cells in MLNs, the following fluorescence-labeled antibodies were used: anti-MHC II IA/E (M5/114.15.2, eBioscience); anti-CD103 (M290, BD Pharmingen); anti-IL-10 (JESS-16E3, BDHorizon); anti-CD64a and b (x54-5/7.1, BD Pharmingen); antiCD45.2 (104, BD Pharmingen); antiCD11b (M1/70, BD Pharmingen) and anti-CD11c (HL3, BD Pharmingen).

Statistics. The Mann Whitney test unpaired Student $t$ test was used to evaluate differences between two experimental groups after checking for normal distribution of data. Statistical significance of Kaplan-Meier survival curves was determined with Log-rank (Mantel-Cox) Test. Significant differences between groups are indicated $\left({ }^{*} P<0.05\right.$; ${ }^{* *} P<0.01$; $\left.{ }^{* * *} P<0.001\right)$. Statistical analysis was performed using GraphPad Prism software (Graphpad Software Inc). In experimental colitis models, body weight changes and stool consistency scores over time were compared between the two treatments (PP versus VGX or WT versus $\mathrm{KO}$ ) using a multivariate linear model for longitudinal measurements (SAS-procedure PROC MIXED) with a heterogeneous autoregressive (firstorder) covariance structure. The choice for the covariance structure was based on the Akaike information criterion (AIC). The least-squares means for each combination of time and treatment were calculated and $p$ values for the comparison of the groups at each point in time were reported after Bonferroni-Holm correction. Analyses were performed using SAS version 9.4 (SAS Institute,).

All supplementary materials are available online at www.molmed.org.

\section{RESULTS}

\section{Vagotomy Impairs Oral Tolerance Most Likely by Reduced Induction of Antigen-specific Regulatory T cells}

As we previously described, vagal input reaches the muscularis externa and activates cholinergic enteric neurons. There, resident muscularis M $\varphi s$ reside in the close vicinity of cholinergic enteric neurons (6). Of note, we observed that also in the mucosa, $\mathrm{CX}_{3} \mathrm{CR}_{1}^{+} \mathrm{M} \varphi$ s are located near choline acetyltransferase (ChAT) positive enteric neuronal fibers (Supplementary Figure S1A, S1B). This suggested that the vagus nerve might also influence intestinal mucosal immune cells via activation of $\mathrm{ChAT}^{+}$enteric neuronal fibers. To investigate vagal modulation of immune cells in the mucosa, we induced oral tolerance in VGX and PP alone mice (Figure 1A). Generally, subcutaneous (s.c.) injection of ovalbumin (OVA) in the footpad of immunized mice results in footpad swelling (that is, inflammation). Oral feeding with OVA in PP alone mice prevented this inflammatory response (Figure 1B), indicative of established oral tolerance. However, OVA feed mice subjected to VGX developed footpad swelling to the same extent as in PBS feed VGX mice (Figure 1B), revealing loss of oral tolerance in mice that underwent VGX. As Tregs are the main players in establishing oral tolerance, we next evaluated the effect of VGX on the development of Tregs in the lamina propria and MLNs via a T cell conversion assay (Figure 1C). Hadis et al. have described that after migrating to the lamina propria, antigen-specific Tregs proliferate, as indicated by an increase in 
A

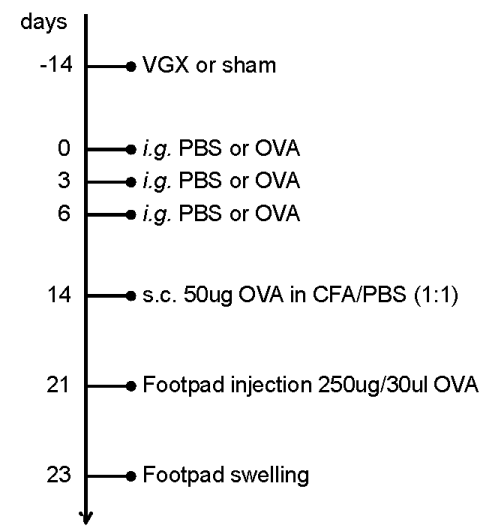

B

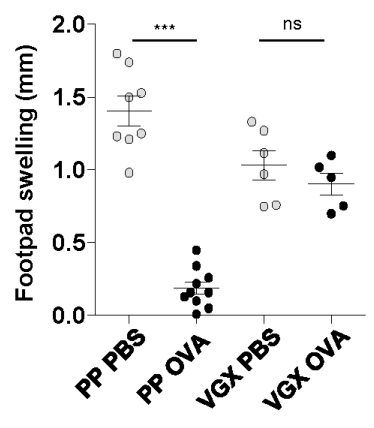

C

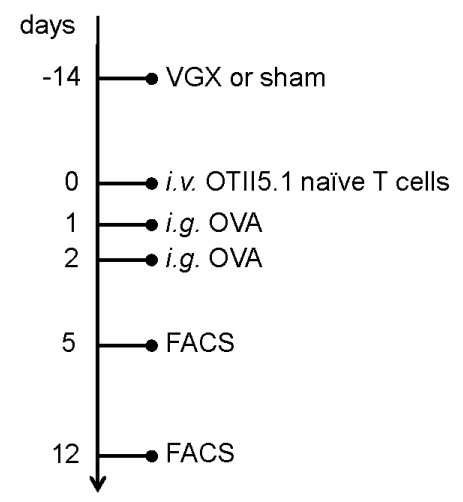

D
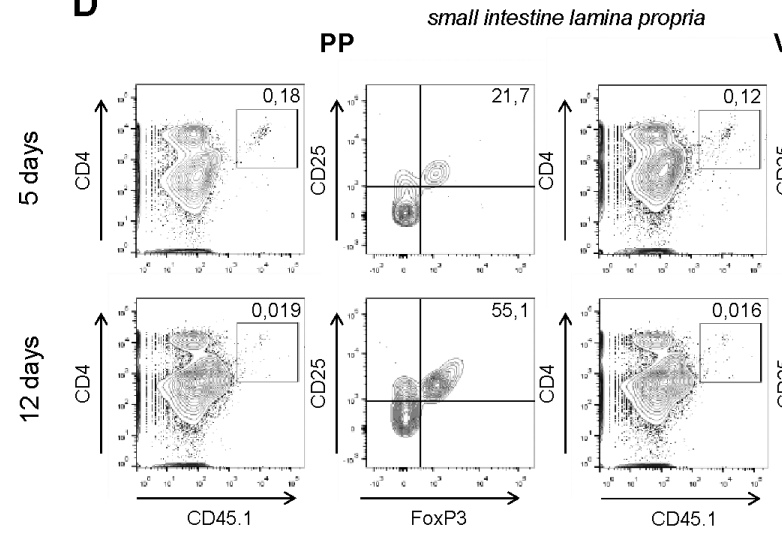

E

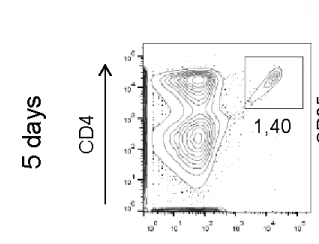

PP
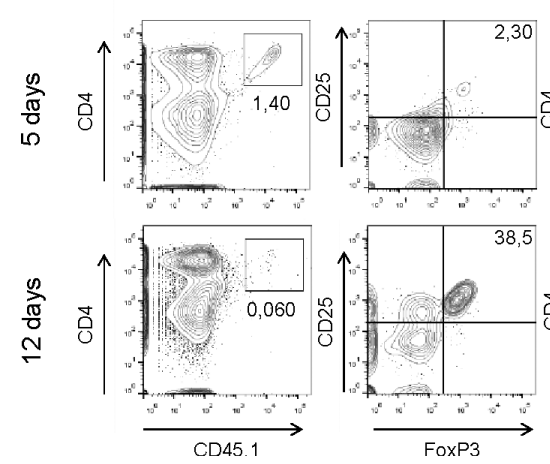

5
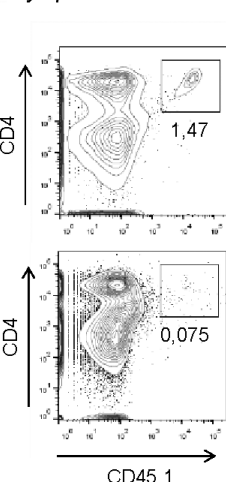

VGX
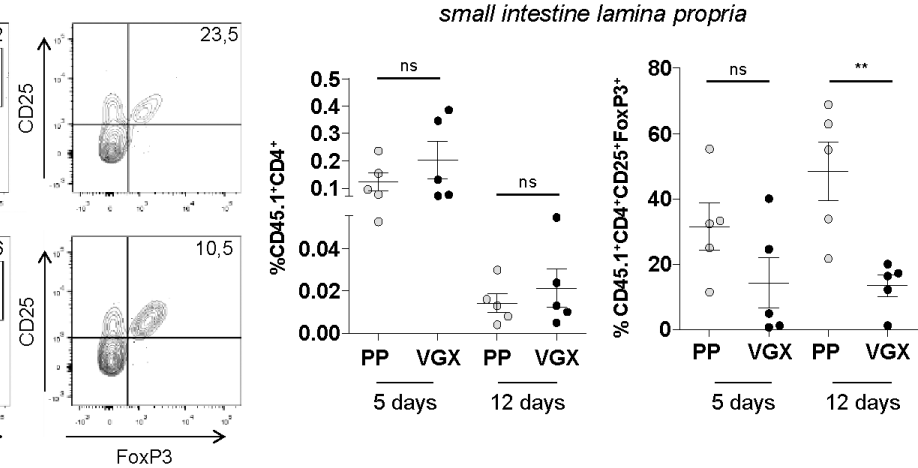

VGX
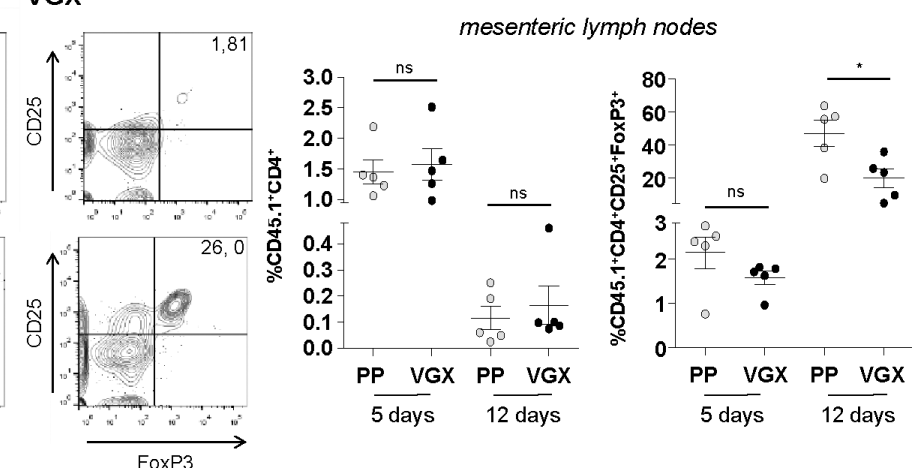

Figure 1. Vagotomy affects induction of oral tolerance and accumulation of antigen-specific regulatory $T$ cells in the lamina propria. (A) Wild type C57BL/6 mice were subjected to vagotomy (VGX) or pyloroplasty (PP) two weeks before 3 intragastric gavages (i.g.) of ovallbumin (OVA) to induce oral tolerance. After 1 wk, all mice were subcutaneously (s.c.) immunized against OVA in CFA and challenged in the footpad $7 \mathrm{~d}$ later with heat-aggregated OVA. Footpad swelling was measured $48 \mathrm{~h}$ after OVA challenge by foodpad injection. Footpad swelling was calculated as difference between prior to and $48 \mathrm{~h}$ after injection of OVA. (B) Footpad swelling was calculated in mice receiving 3 intragastric gavages (i.g.) of PBS or OVA and subjected to VGX or PP. In PP alone mice the reduction in footpad swelling is indicative of establishment of oral tolerance while VGX failed to develop oral tolerance. (C) OT-II naiive CD4 T cells were adoptively transferred into syngeneic VGX and PP recipients before two oral doses of $50 \mathrm{mg}$ OVA. Conversion and accumulation of OT-ll naïve CD4 T cells into Tregs were analyzed 5 and $12 \mathrm{~d}$ after adoptive transfer. Representative dot plot of transferred OTII T cells (CD45. $1^{+} \mathrm{CD} 4^{+}$cells) and Tregs $\left(\mathrm{CD} 45.1^{+} \mathrm{CD} 4^{+} \mathrm{CD} 25^{+} \mathrm{FoxP} 3^{+}\right.$cells) generated out of adoptively transferred OT-II CD45. $1^{+} \mathrm{CD} 4^{+}$cells and frequencies in small intestine lamina propria (D) and MLNs (E) are displayed. Circles represent individual mice. Data are representative of two independent experiments. Statistical significance was determined with unpaired Student $t$-test $\left({ }^{*} P<0.05,{ }^{* *} P<0.01,{ }^{* * *} P<0.001\right.$, ns, not significant). 
Tregs at $\mathrm{d} 12$ compared with $\mathrm{d} 5$ following oral antigen exposure (23). Based on this study, OVA-specific naïve T cells isolated from OT-II mice were adoptively transferred into syngeneic VGX and PP recipient mice to evaluate the conversion and accumulation of OVA-specific Tregs at d 5 and 12 following exposure to OVA in both VGX and PP groups (Figure 1C). FACS analysis of single cell suspensions isolated from the small intestine lamina propria (Figure 1D) and MLNs (Figure 1E) of VGX mice revealed a significantly lower percentage of OVA-specific Tregs $\left(\mathrm{CD} 45.1^{+} \mathrm{CD}^{+} \mathrm{CD}^{2} 5^{+} \mathrm{FoxP}^{+}\right.$) (Figure 1D, E) compared with cell fractions isolated from PP alone mice at $12 \mathrm{~d}$, while no differences were observed on the resident T cells (Supplementary Figure S2). These data suggest that an intact vagal innervation is crucial for the establishment of oral tolerance and for the conversion of naïve mucosal T cells into Tregs.

\section{Vagotomy Worsens the Development of Acute Dextran Sulphate Sodium (DSS)-Induced Colitis But Not That of Chronic T Cell Transfer-induced Colitis}

Based on the above, we next investigated the effect of VGX on two different models of colitis, that is, DSS colitis and $\mathrm{T}$ cell transfer model of chronic colitis.

DSS colitis. To investigate the effects of VGX on the development of colitis, mice underwent PP alone or in combination with VGX. Both groups were treated with $2 \%$ DSS in drinking water. Both groups lost body weight from d 5 onwards and showed alterations in stool consistency from d 4. As expected, VGX mice lost more body weight than PP alone mice, but differences only reached statistically significance at d 10, 11, 12,13 and 14 after DSS treatment started (Figure 2A). In addition, VGX mice presented significant higher stool consistency score compared with PP alone mice (Figure 2B).

To evaluate the degree of inflammation in VGX and PP mice during the acute phase of colitis, a set of animals was sacrificed at d 7. At this time point, PP and VGX mice showed similar colon length, spleen weight (Supplementary
Figure S3A) and proinflammatory cytokine gene expression. However, il1a and tbet reach significantly higher expression levels in VGX mice (Supplementary Figure S3B). Interestingly, colonic $\mathrm{CD}^{+} \mathrm{T}$ cells were significantly increased (PP 5,7\% $\pm 0,7 \%$ versus VGX $\left.8 \% \pm 1.1 \% ;{ }^{*}, P=0,0057\right)$, while Tregs were decreased in VGX mice (VGX $3.4 \% \pm 0,3 \%$ versus PP6, $2 \% \pm 0,6 \%$; *, $P=0,0239)$, confirming that VGX mice developed more severe inflammation (Figure 2D). In addition, flow cytometric characterization of $\mathrm{CD}^{+} 4^{+}$cells that have migrated into MLNs revealed that $\mathrm{MCHII}^{+} \mathrm{IL} 10^{+} \mathrm{CD} 64^{+}$cells were significantly reduced in VGX compared with PP mice (Figure 2E). Interestingly, differences observed at $\mathrm{d} 7$ were even more pronounced during the recovery phase of colitis (assessed at d 14). In fact, PP mice were able to recover faster compared with VGX mice, regaining body weight $(95.45 \pm 4.60 \%$ at $\mathrm{d} 14)$ and lowering the stool consistency score (1.25 \pm 0.37 value at $\mathrm{d} 14)$. On the contrary, mice subjected to VGX presented more severe colitis and even failed to survive the DSS treatment (Figure 2C). Altogether, these data demonstrate that VGX worsens DSS-induced colitis during both the acute and the recovery phase.

$\mathrm{T}$ cell transfer colitis. WT $\rightarrow$ VGX-Rag1 and $\mathrm{WT} \rightarrow$ PP-Rag1 mice developed equal disease severity with comparable body weight loss (Figure 3A). Moreover, 8 wks after $\mathrm{T}$ cell transfer both groups presented comparable colon length and spleen weight (Figure 3B). Accordingly, no significant differences in gene expression of proinflammatory cytokines (tnfa, Il6, Il1a, tbet, infg, rorc and Il17a) were measured in colonic tissue between $\mathrm{WT} \rightarrow$ VGX-Rag1 and $\mathrm{WT} \rightarrow$ PP-Rag1 mice (Figure 3C). Eventually, using flow cytometry, we showed that the proportion of proinflammatory $\mathrm{T}$ cells (that is, T helper cells: IFN- $\gamma^{+}$producing T cells, Th1; and IL17-A ${ }^{+}$producing T cells, Th17) and Tregs $\left(\mathrm{CD} 4^{+} \mathrm{CD} 25^{+} \mathrm{FoxP}^{+}\right)$was similar in both groups (Figure 3D). Thus, our data showed that unlike DSS colitis, VGX did not worsen chronic colitis induced via $\mathrm{T}$ cell transfer.

\section{Conversion of Antigen-specific Regulatory T Cells and Induction of Oral Tolerance Are Independent of $\alpha 7 n A C h R$}

As the previous experiments revealed an important role of the vagal innervation in oral tolerance and DSS colitis, we further investigated the potential role of $\alpha 7 \mathrm{nAChR}$. To this end, oral tolerance was induced in $\alpha 7 \mathrm{nAChR}^{-/-}$and $\alpha 7 \mathrm{nAChR}^{+/+}$ mice. Oral gavage of OVA prevented footpad swelling to the same extent in both $\alpha 7 \mathrm{nAChR}^{-/-}$and $\alpha 7 \mathrm{nAChR}^{+/+}$ mice assessed $48 \mathrm{~h}$ after s.c. injection of OVA (Figure 4A). Moreover, the ability to convert antigen-specific naïve $T$ cells into antigen-specific Tregs was evaluated by flow cytometry analysis of T cell subsets in the small intestine lamina propria and MLNs. Frequencies of OVA-specific naïve T cells isolated from $\alpha 7 \mathrm{nAChR}^{-/-}$and $\alpha 7 \mathrm{nAChR}^{+/+}$mice were similar in the small intestine lamina propria (Figure 4B) and MLNs (Figure 4C). In addition, the frequency of OVA-specific Tregs in the small intestine lamina propria and MLNs was similar in both groups (Figure 4B, C), indicating that $\alpha 7 \mathrm{nAChR}$ does not affect the conversion of naïve T cells into Tregs. Consistently, $\alpha 7 \mathrm{nAChR}{ }^{-/-}$mice showed no differences in the ability of resident $\mathrm{T}$ helper cell to polarize into Tregs in the small intestine lamina propria (Supplementary Figure S4), suggesting that $\alpha 7 \mathrm{nAChR}$ is not crucial for the polarization of naïve $T$ cells into $\mathrm{T}$ regulatory.

\section{Development of Both Acute (DSS) and Chronic (T Cell Transfer) Colitis is Independent of $\alpha 7 \mathrm{nAChR}$}

DSS colitis. Both $\alpha 7 \mathrm{nAChR}^{+/+}$and $\alpha 7 \mathrm{nAChR}^{-/-}$mice presented similar severity of DSS colitis, as shown by comparable body weight loss and stool consistency score (Figure 5A, B). The severity of colitis was highest for both groups on d $8\left(\alpha 7 \mathrm{nAChR}^{+/+} 87,6 \% \pm 6,6 \%\right.$ versus $\alpha 7 \mathrm{nAChR} \mathrm{Ch}^{-/-} 90,6 \% \pm 6,9 \%$ of initial body weight, Figure 5A) after which mice recovered in the following days. On d 14, colon length and spleen weight (Figure 5C) as well as proinflammatory gene expression (Figure 5D) were not significantly 
A

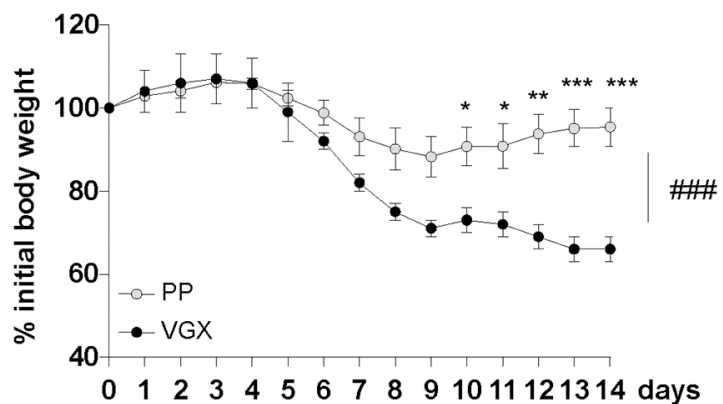

B

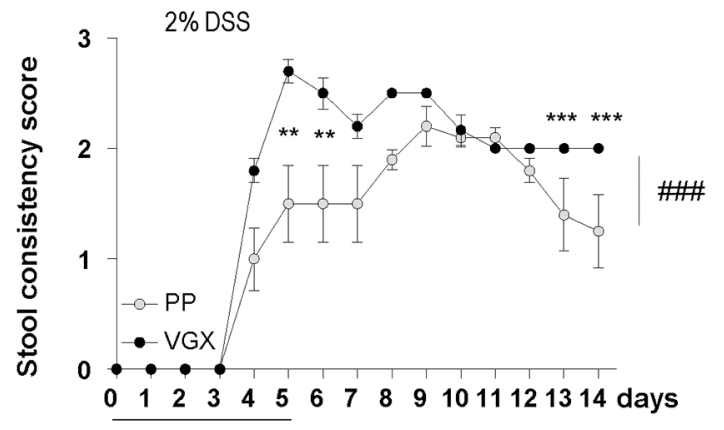

C

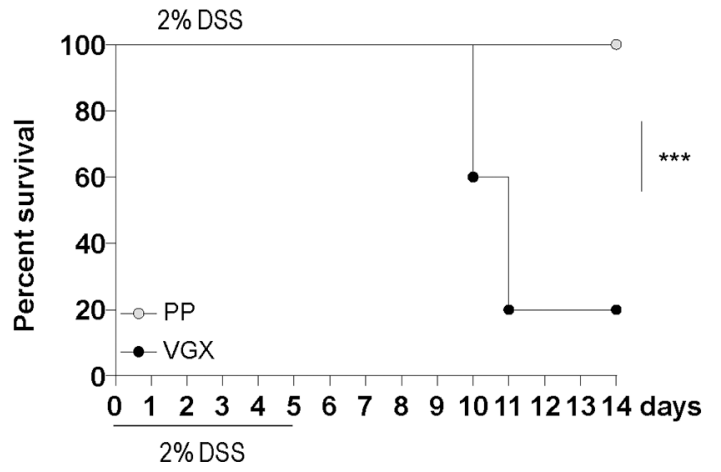

D
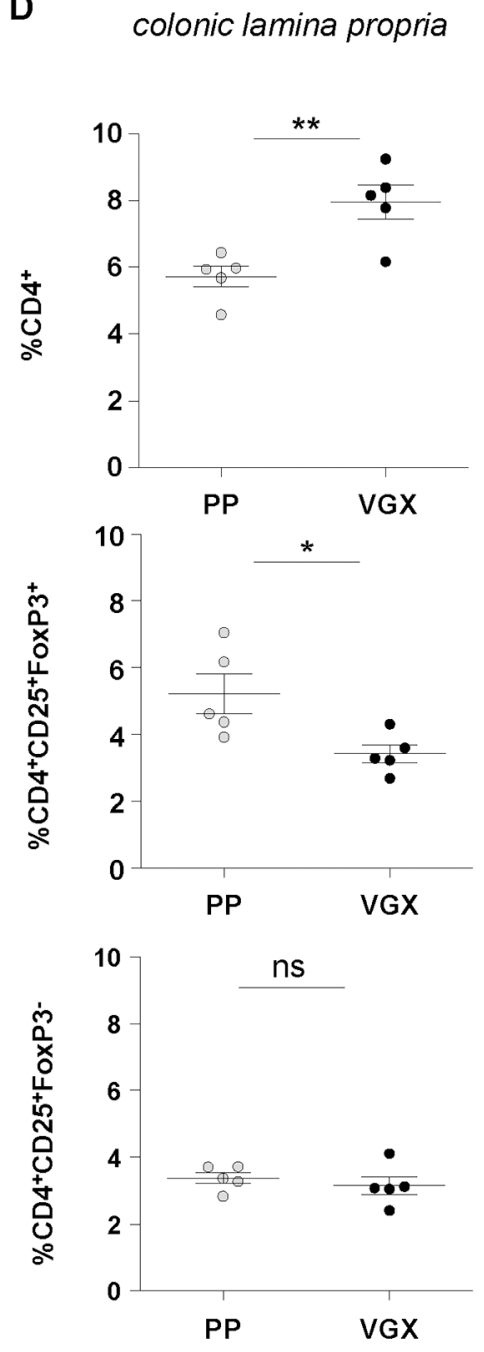

E

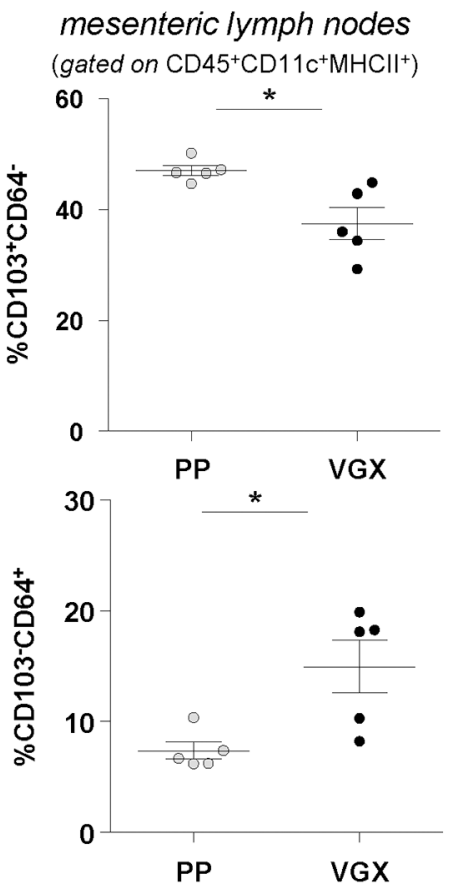

(gated on $\mathrm{CD} 45^{+} \mathrm{CD} 11 \mathrm{~b}^{+} \mathrm{CD} 64^{+}$)

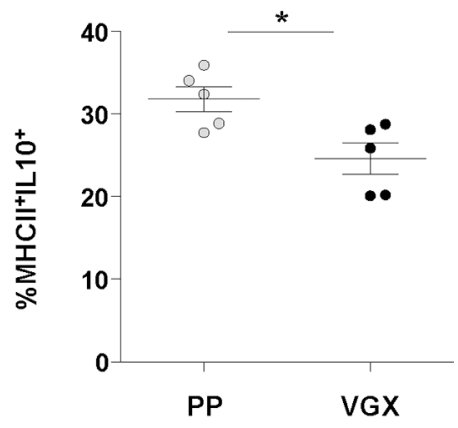

Figure 2. Vagotomy worsens the severity of DSS-induced colitis. Wild type C57BL/6 mice were subjected to vagotomy (VGX) or pyloroplasty (PP) 2 wks before treatment with 2\% DSS in drinking water for $5 \mathrm{~d}$ followed by $9 \mathrm{~d}$ of normal drinking water. Body weight (A) and stool consistency score (B) were assessed daily. Statistical significance was determined using a multivariate linear model after a post-hoc Bonferroni-Holm correction (\#\#\# $P<0.001$, ${ }^{*} P<0.05$, ${ }^{* *} P<0.01,{ }^{* * *} P<0.001$ ). (C) Mortality is expressed as survival rate and shown by Kaplan-Meier survival curves. Data are representative of two independent experiments. Statistical significance of Kaplan-Meier survival curves was determined with Log-rank (Mantel-Cox) Test (***, $p=0.0004$ ). At d 7 after induction of DSS-colitis, PP and VGX mice were sacrificed. Colonic lamina propria immune cells were characterized by flow cytometry. (D) Frequencies of colonic CD4 ${ }^{+} \mathrm{T}$ cells, CD25 $5^{+}$OxP3 $^{+}$Tregs and CD25 FoxP3- T cells are shown. (E) Frequencies of innate immune cells, CD $103^{+}$and CD64 $^{+}$antigen presenting cells isolated from the MLNs. Dots represent frequencies of immune cells in individual mice. Mean \pm SEM is also shown. Data are representative of two independent experiments. Statistical significance was determined with the unpaired Student $t$-test $\left({ }^{*} P<0.05\right.$, ${ }^{* *} P<0.01 ;$ ns, not significant).

different between $\alpha 7 \mathrm{nAChR}{ }^{+/+}$and $\alpha 7 \mathrm{nAChR} R^{-/-}$mice. These data indicate that $\alpha 7 \mathrm{nAChR}$ signaling does not play a major role in the establishment of the vagal anti-inflammatory effect during DSS colitis.
$\mathrm{T}$ cell transfer colitis. To unravel the role of $\alpha 7 \mathrm{nAChR}$ on $\mathrm{T}$ cells during colitis, Rag $1^{-/-}$mice were injected with naïve $\mathrm{T}$ cells isolated from the spleen of $\alpha 7 \mathrm{nAChR}^{+/+}$or $\alpha 7 \mathrm{nAChR}^{-/-}$mice using cell sorting. As shown in Figure 6A, both groups showed body weight loss with a similar time course and severity. Moreover, 10 wks after $\mathrm{T}$ cell adoptive transfer, colon length and spleen weight were not significantly different (Figure 6B). In line, inflammatory cytokine gene 
A

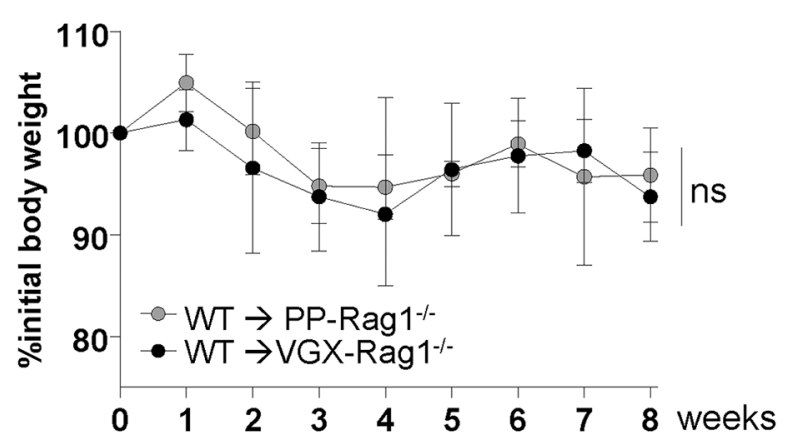

D

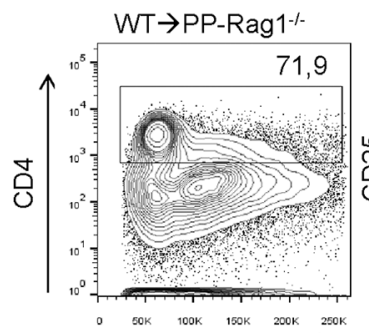
WT $\rightarrow$ VGX-Rag ${ }^{100 k}$
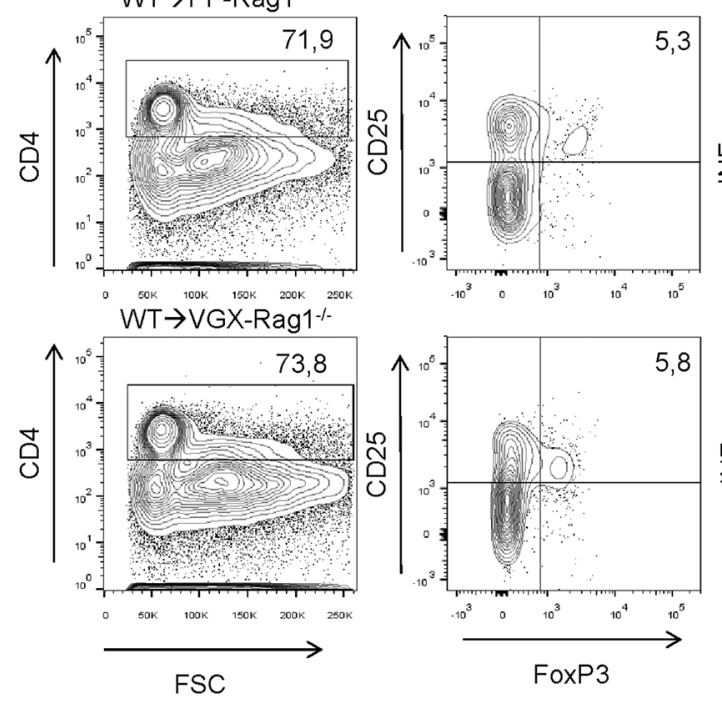

B

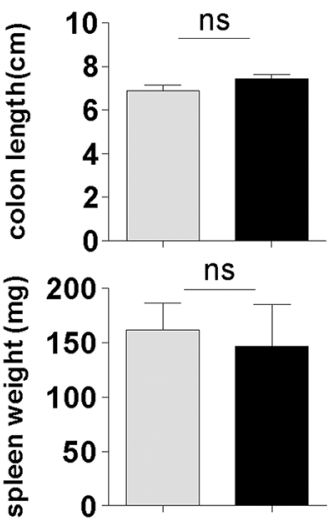

C

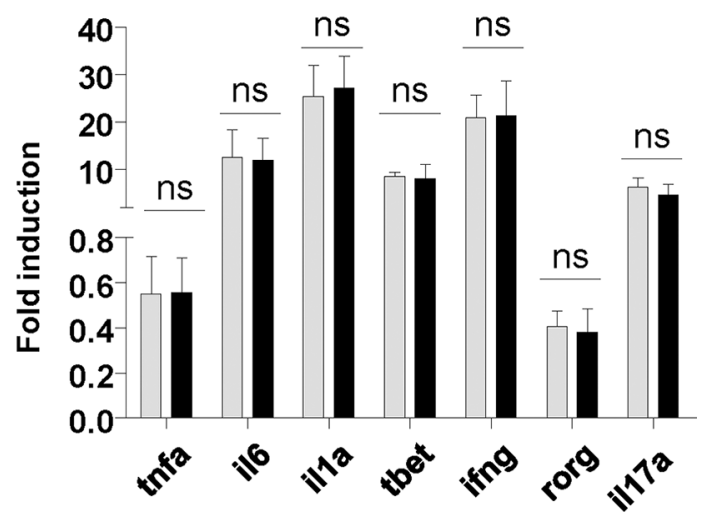

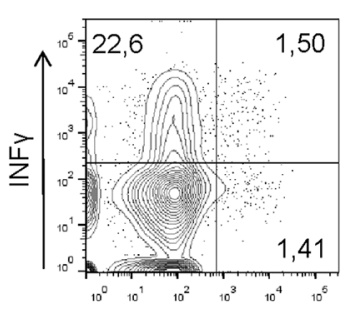
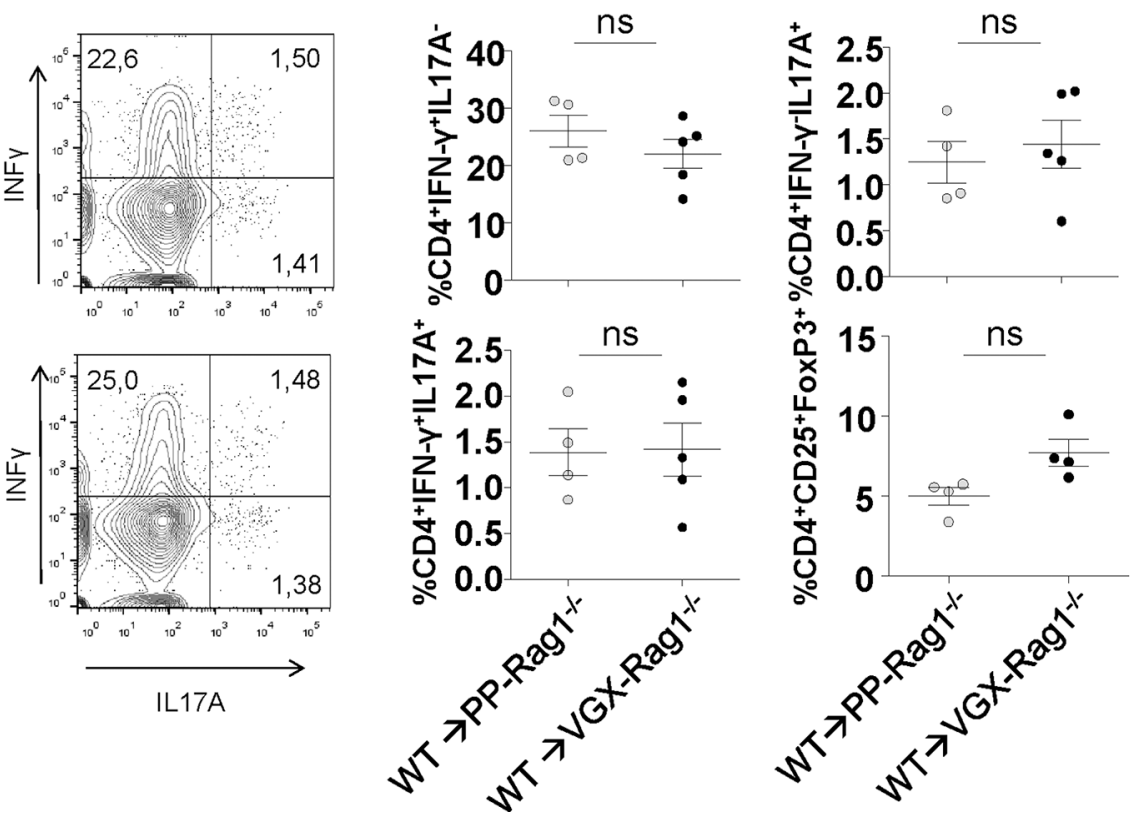

Figure 3. Vagotomy does not affect the development and the severity of T cell transfer-induced chronic colitis. Rag ${ }^{-/-}$mice were subjected to vagotomy (VGX) or pyloroplasty (PP) 2 wks before adoptive transfer of naïve T cells. (A) Body weight loss was followed over time in VGX-Rag $1^{-1-}$ mice injected with WT naïve T cells (WT $\rightarrow$ VGX-Rag 1, black) and in PP-Rag ${ }^{-1-}$ mice injected with WT naïve T cells $(\mathrm{WT} \rightarrow \mathrm{PP}-\mathrm{Rag} \mathrm{l}$, gray). Statistical significance was determined using a multivariate linear model after a post-hoc Bonferroni-Holm correction (ns, not significant). (B) Eight weeks after T cell transfer, colon length and spleen weight were measured in WT $\rightarrow$ VGX-Rag 1 and WT $\rightarrow$ PP-Rag 1 mice. (C) Inflammatory gene expression was quantified via q-PCR in colonic tissue. thfa, il6, illa, tbet, infg, rorc and ill7a mRNAs were analyzed. (C) Gene expression levels are shown as fold induction on non-injected naïve Rag- ${ }^{1 /}$. (D) Representative flow

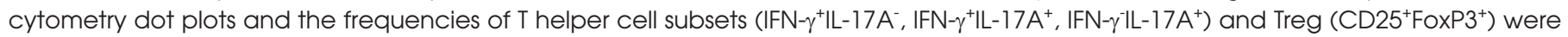
showed. Dots represent individual mice. Data are expressed as mean \pm SEM. Statistical significance was determined with the unpaired Student t-test for colon length, spleen weight, gene expression level and flow cytometry analysis (ns, not significant).

expression (tnfa, Il6, Il1a, tbet, infg, rorc and $I l 17 a)$ was similar in $\alpha 7 \mathrm{nACh}-$

$\mathrm{R}^{+/+} \rightarrow$ Rag1 and $\alpha 7 \mathrm{nAChR}{ }^{-/-} \rightarrow$ Rag1 mice (Figure 6C). Finally, flow cytometric analysis of colonic single cell suspensions revealed equal proportion of Th1 and Th17 and Tregs in the colonic lamina propria (Figure 6D) and MLNs (data not shown). Overall, these data indicate that the absence of $\alpha 7 \mathrm{nAChR}$ on $\mathrm{T}$ cells does not influence $\mathrm{T}$ cell conversion, $\mathrm{T}$ cell proliferation and expansion in the gut during inflammation.

To evaluate whether other cells than $\mathrm{T}$ cells may be modulated through 
A

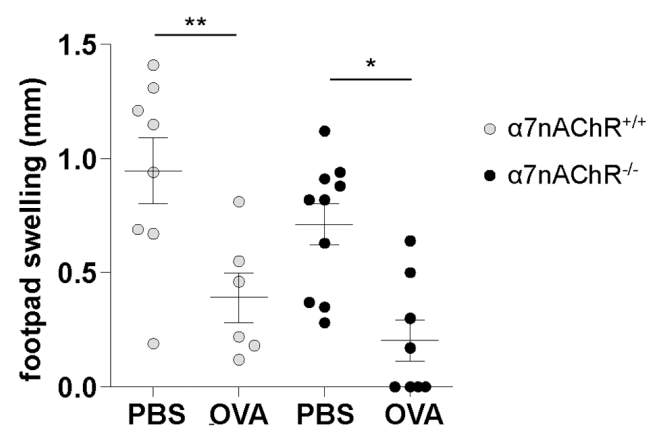

B
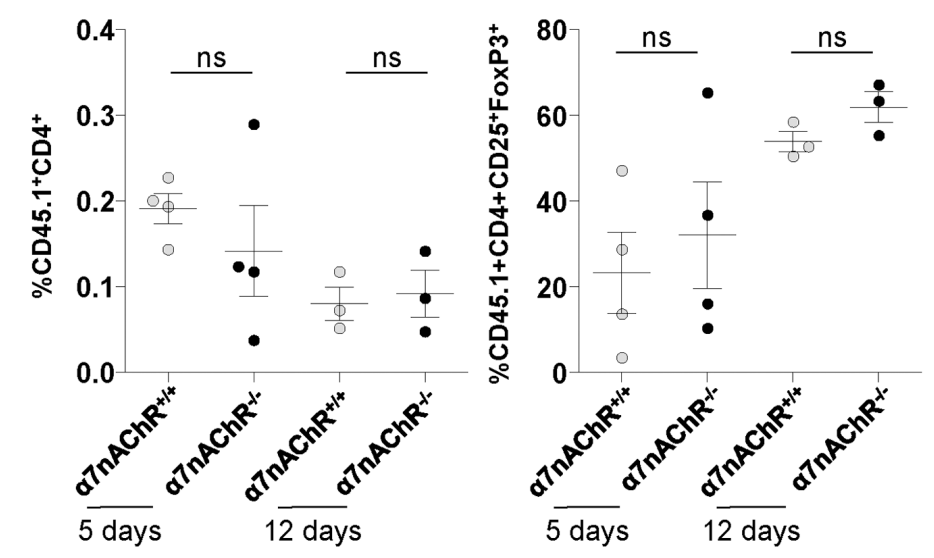

C

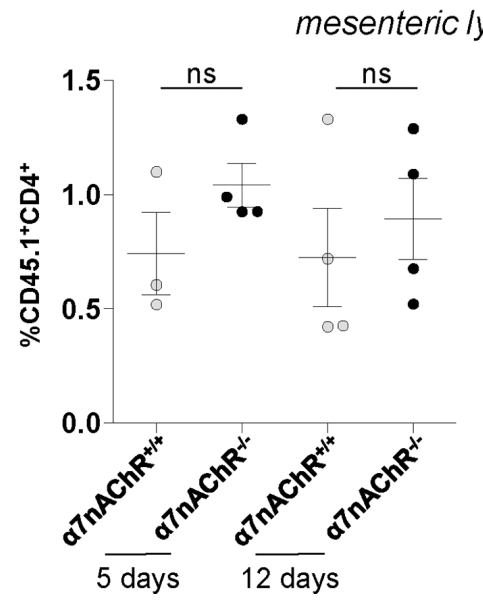

ymph nodes

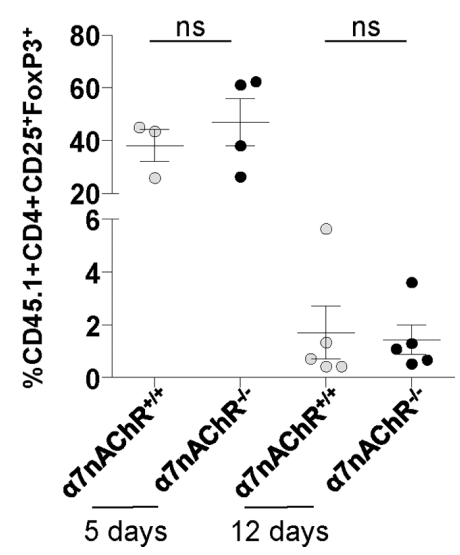

Figure 4. Oral tolerance and antigen-specific regulatory $T$ cell induction are independent of $\alpha 7 n A C h R$. Oral tolerance was induced in $\alpha 7 n \mathrm{AChR}^{+/+}$and $\alpha 7 n \mathrm{AChR}^{-/-}$mice with oral gavage of OVA or PBS as control. (A) OVA-specific delayed-type hypersensitivity response was determined by footpad swelling $48 \mathrm{~h}$ after OVA challenge. OT-II T cells were adoptively transferred into $\alpha 7 n \mathrm{AChR}^{+/+}$and $\alpha 7 \mathrm{nAChR}{ }^{-/-}$recipients and 5 and $12 \mathrm{~d}$ after adoptive transfer, Treg population was evaluated by flow cytometry. Plots are gated on CD45. $1^{+} \mathrm{CD} 4^{+}$cells. The frequency of $\mathrm{CD} 25^{+} \mathrm{FoxP}^{+}$Tregs generated out of adoptively transferred OVA-specific CD45. $1^{+} C D 4^{+}$cells was determined in small intestine lamina propria (B) and MLNs (C). Dots represent individual mice. Data are expressed as mean \pm SEM. Data representative of two independent experiments. Statistical significance was determined with unpaired Student t-test $\left({ }^{*} P<0.05,{ }^{* *} P<0.01\right.$; ns, not significant). the vagus, we studied the impact of $\alpha 7 \mathrm{nAChR}$ expression in recipient Rag $1^{-1-}$ mice. To this end, we generated $\alpha 7 \mathrm{nAChR}^{-/-} \operatorname{Rag} 1^{-/}$mice and adoptively transferred naïve $\mathrm{T}$ cells from $\mathrm{WT}$ C57BL $/ 6 \mathrm{~J}$ mice into $\alpha 7 \mathrm{nAChR}{ }^{-/} \operatorname{Rag} 1^{-/-}$ and their $\alpha 7 \mathrm{nAChR}^{+/+} \mathrm{Rag}^{-/-}$littermates. Both groups of mice underwent similar weight loss over time (Figure 7A). Five weeks after $\mathrm{T}$ cell adoptive transfer, mice were sacrificed and disease severity was assessed. Both groups showed similar colon length and spleen weight (Figure 7B). Gene expression levels of proinflammatory cytokines in the colon were comparable between WT $\rightarrow$ $\alpha 7 \mathrm{nAChR}^{-/} \mathrm{Rag1}^{-/-}$and WT $\rightarrow \alpha 7 \mathrm{nACh}-$ $\mathrm{R}^{+/+} \mathrm{Rag}^{-/-}$(Figure 7C). In addition, the percentage of $\mathrm{T}$ helper cells and Tregs in the colonic lamina propria (Figure 7D) and MLNs (data not shown) were not significantly different, suggesting that $\alpha 7 \mathrm{nAChR}$ is not involved in the endogenous vagal modulation of intestinal inflammation.

\section{DISCUSSION}

Although the interaction between the vagus nerve and the immune system has been extensively studied, the exact mechanism leading to an increased susceptibility to develop colitis following VGX still needs to be clarified (4). Here, we investigated the possible impact of VGX on oral tolerance, the conversion process of Tregs cells and the development of colitis. We showed that VGX disturbs the establishment of oral tolerance, interfering with the induction and expansion of Tregs in the MLNs and in the intestinal lamina propria. Moreover, we confirmed that reduction of Tregs conversion in VGX-mice was associated with an increased susceptibility to develop acute DSS-induced acute colitis, but did not alter the course of $\mathrm{T}$ cell transfer-induced chronic colitis. Of note, absence of $\alpha 7 \mathrm{nAChR}$ failed to affect the development of both oral tolerance and colitis. Based on these data, we concluded that the endogenous vagal input to the intestinal mucosal immune system plays an important role in the induction 
A

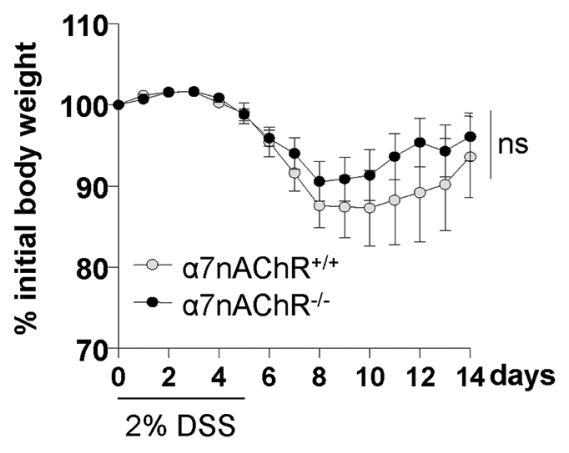

C
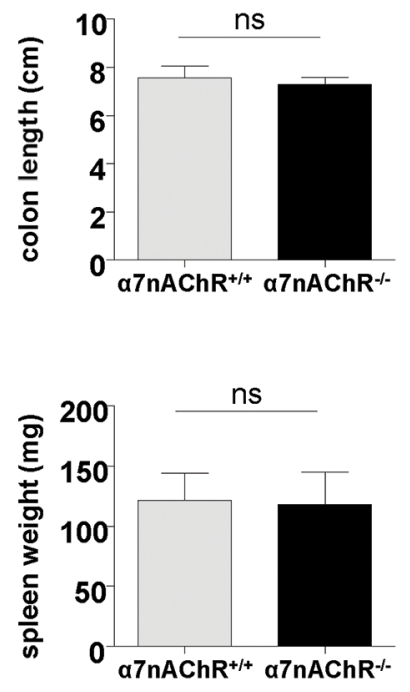

D
B
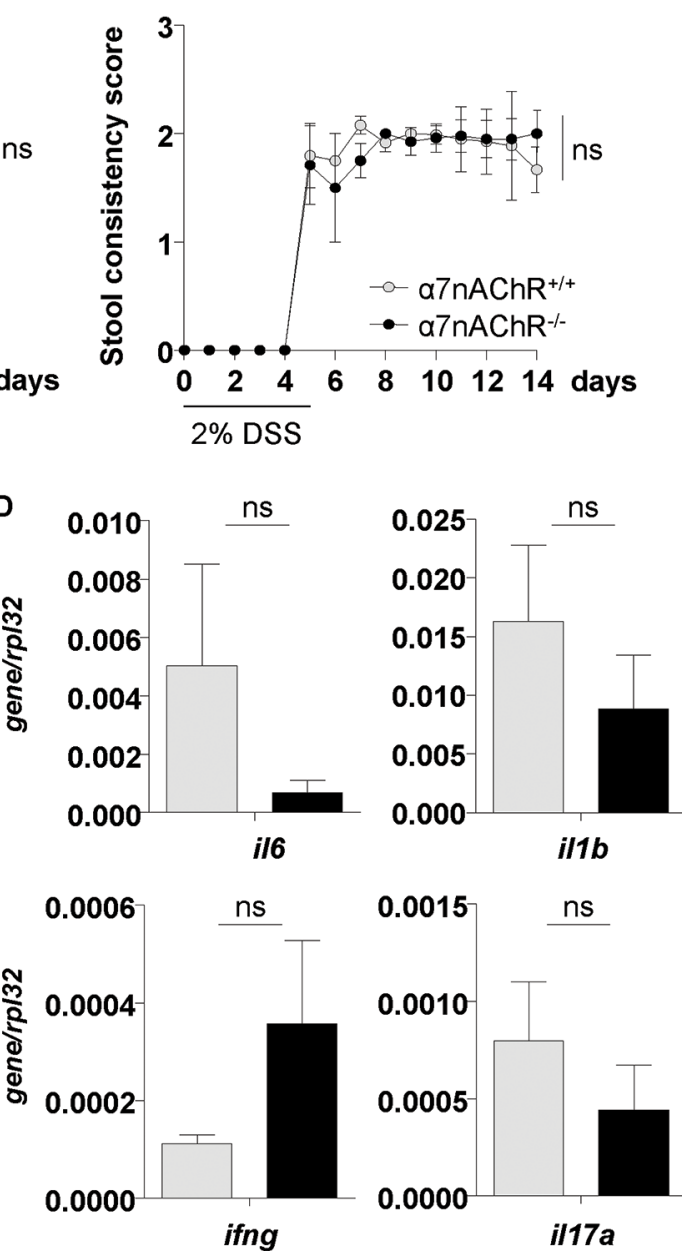

Figure 5. $\alpha 7 n A C h R$ expression does not influence the severity of acute DSS colitis. $\alpha 7 \mathrm{nAChR}^{-/}$and $\alpha 7 \mathrm{nAChR} \mathrm{R}^{+/+}$mice were subjected to DSS-colitis. (A) Body weight and (B) stool consistency were scored daily. Statistical significance was determined using a multivariate linear model after a post-hoc Bonferroni-Holm correction for body weight changes and stool consistency score (ns, not significant). (C) Fourteen days after DSS treatment, mice were sacrificed to measure colon length and spleen weight. (D) Inflammatory gene expression was quantified via q-PCR in colonic tissue. Gene expression levels are shown relative to rp/32 mRNA expression. Data expressed as mean \pm SEM, are representative of three independent experiments. Statistical significance was determined with the unpaired Student $t$-test for colon length, spleen weight, gene expression level and flow cytometry analysis (ns, not significant).

of oral tolerance and acute colitis in a $\alpha 7 n A C h R$-independent manner.

Installation of oral tolerance implicates active regulatory processes including the induction of antigen-specific Tregs. Tregs are essential to prevent immune responses against food and commensal antigens (24). In the GI tract, expansion of antigen-specific Tregs is tightly regulated by the lamina propria microenvironment. As shown by Hadis et al. (23), oral tolerance is initiated in the MLNs where mucosal DCs generate a pool of FoxP3 ${ }^{+}$ Tregs from antigen-specific naive $\mathrm{T}$ cells. Subsequently, Tregs migrate to the intestinal lamina propria where proliferate. Of note, antigen-presenting cells identified as the $\mathrm{CX}_{3} \mathrm{CR}_{1}^{+}$macrophages support survival and proliferation of the incoming Tregs by releasing high amounts of IL-10. In fact, $\mathrm{CX}_{3} \mathrm{CR}_{1}$-deficient mice show reduced production of IL-10 in the lamina propria, and a reduced number of intestinal Tregs at $12 \mathrm{~d}$ following oral exposure to OVA and thus fail to develop oral tolerance (23). Based on this study, we evaluated the number of Tregs at $\mathrm{d} 5$ and 12 following exposure to OVA in VGX and PP mice. Here, we show for the first time that VGX impairs the ability to develop oral tolerance, a finding that is associated with a reduction in Tregs in the lamina propria and in MLNs. Of note, the proportion of Tregs at d 12 was significantly reduced in both lamina propria and MLNs, suggesting that the survival and/or proliferation of Tregs was compromised in VGX mice. As Hadis et al. showed that $\mathrm{CX}_{3} \mathrm{CR}_{1}^{+} \mathrm{M} \varphi$ s releasing high levels of IL-10 play a major role in this process, one can speculate that the vagal input or cholinergic tone is involved in driving the IL-10 production of these immune cells. This hypothesis is indirectly supported by our finding that, similar to the resident macrophages in the muscularis externa (6), $\mathrm{CX}_{3} \mathrm{CR}_{1}{ }^{+} \mathrm{M} \varphi \mathrm{s}$ are in close proximity of $\mathrm{ChAT}^{+}$neuronal fibers also in the intestinal mucosa. Based on the above, we speculate that VGX affects induction of oral tolerance by primarily reducing conversion of Tregs in the lamina propria and in the MLNs. Further studies will have to clarify whether the reduced cholinergic input to the resident $\mathrm{CX}_{3} \mathrm{CR}_{1}^{+}$macrophages cells or the direct effect of acetylcholine on Tregs is responsible for the alteration in oral tolerance.

In addition to oral tolerance installation, Tregs are also necessary to control and resolve intestinal inflammation thus suggesting that reduce Tregs conversion in VGX mice may predispose to colitis (25). In line with previous studies (8-11, 26), we showed that VGX mice are more susceptible to DSS colitis. During acute DSS colitis, VGX mice lost more body weight and showed higher stool consistency score. Moreover, these mice had less colonic Tregs and showed a decrease in IL-10 expressing $\mathrm{CD}^{+} 4^{+}$macrophages 
A

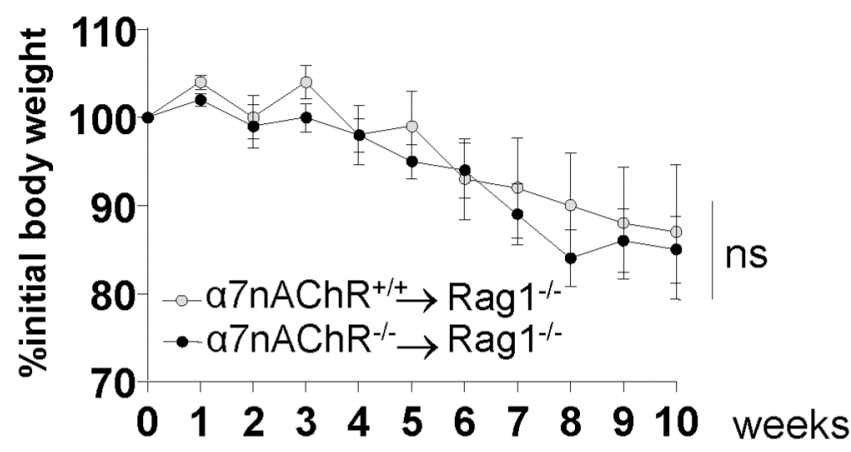

B

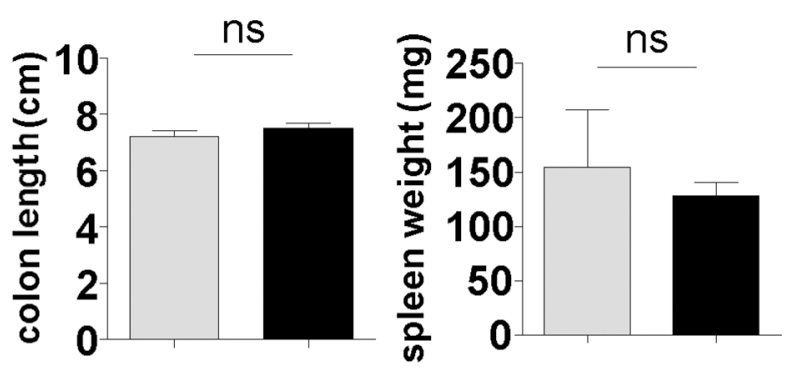

C

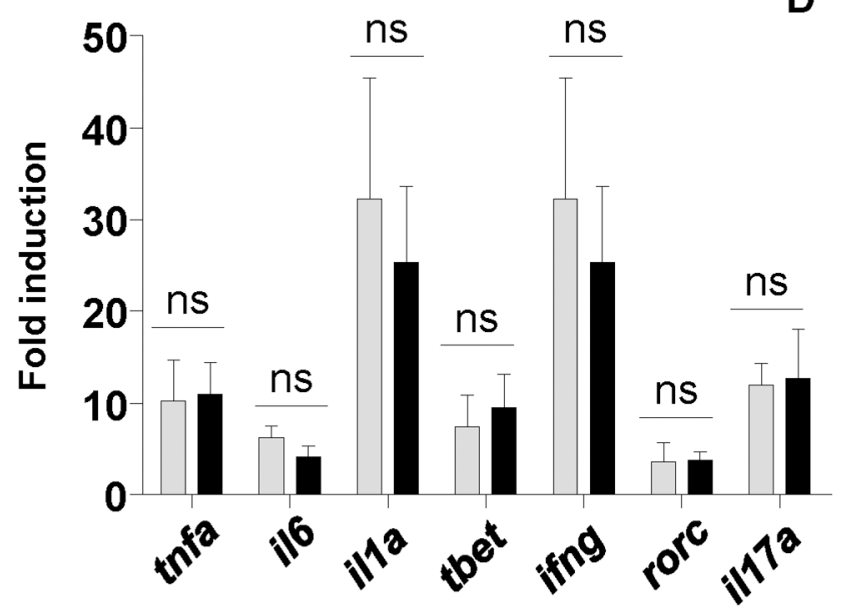

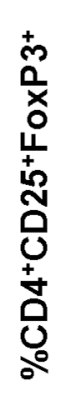

ns

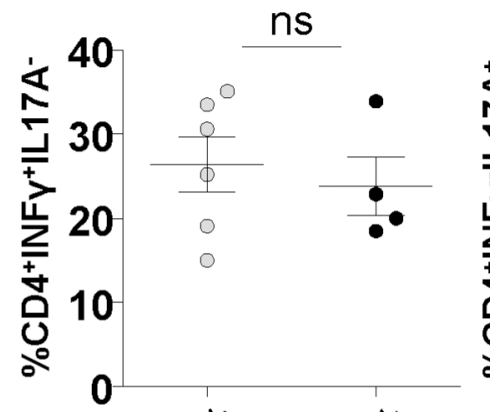

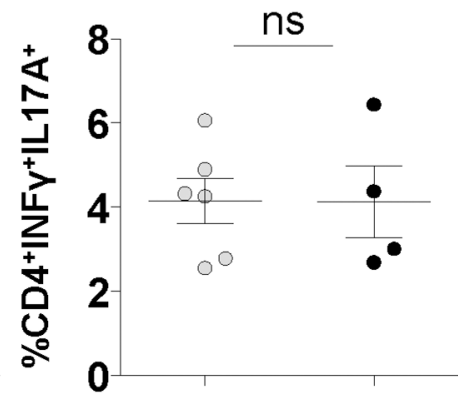

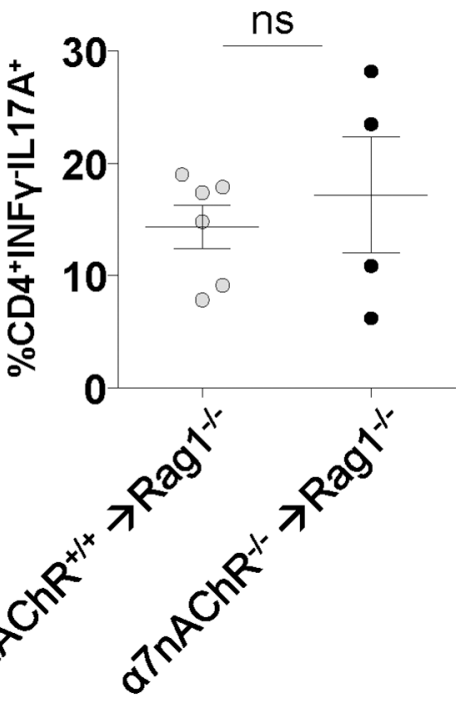

Figure 6. Lack of T cell-specific $\alpha 7 n A C h R$ signaling does not influence severity of T cell transfer induced colitis. Rag ${ }^{-/-}$mice were adoptively transferred with naïve CD4 T cells isolated respectively from $\alpha 7 n A C h R^{+/+}$or $\alpha 7 n A C h R^{-1-}$ mice. (A) Body weight loss was followed over time in Rag $1^{-/}$mice injected with $\alpha 7 n A C h R^{+/+}$naïve T cells ( $\alpha 7 n A C h R^{+/+} \rightarrow$ Rag 1, gray) and in Rag 1/- mice injected with $\alpha 7 n A C h R^{-1-}$ naïve T cells ( $\alpha 7 \mathrm{nAChR}^{-1} \rightarrow$ Rag 1, black). Statistical significance was determined using a multivariate linear model after a post-hoc Bonferroni-Holm correction for body weight changes and stool consistency score (ns, not significant). (B) Ten weeks after T cells transfer, colon length and spleen weight were measured in $\alpha 7 \mathrm{nAChR}^{+/+} \rightarrow \mathrm{Rag} 1$ and $\alpha 7 \mathrm{nAChR}{ }^{-1} \rightarrow$ Rag 1 mice. (C) Inflammatory gene expression was quantified via q-PCR in colonic tissue. tnfa, il6, illa, tbet, infg, rorc and il 17a mRNAs were analyzed. Gene expression levels are shown relative to rp/32 mRNA expression. The frequencies of Th cells isolated from the colonic lamina propria were determined by flow cytometry. Gates were set on CD4 ${ }^{+}$cells. (D) Frequencies of Th cell subsets (IFN- $\gamma^{+} I L-17 A^{-}$, IFN- $\gamma^{+} I L-17 A^{+}$, IFN- $\gamma^{-} I L-17 A^{+}$) and Tregs $\left(C D 25^{+}\right.$FoxP3 ${ }^{+}$). Dots represent individual mice. In addition, data are shown as mean \pm SEM. Data are representative of 3 independent experiments. Statistical significance was determined with the unpaired Student t-test (ns, not significant) for colon length, spleen weight, gene expression level and flow cytometry analysis. 
A

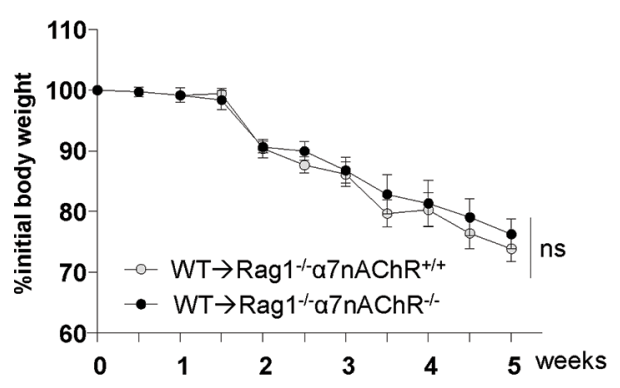

C

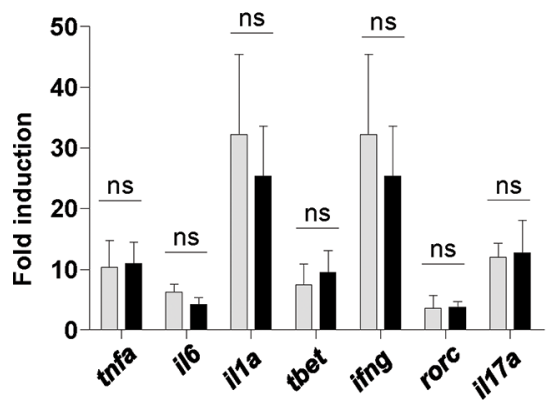

B

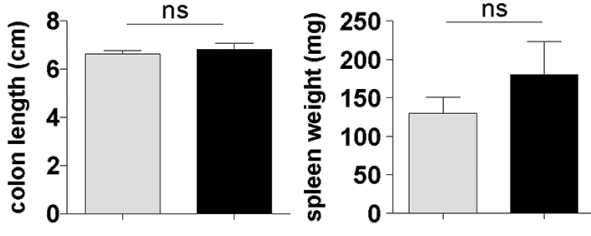

D

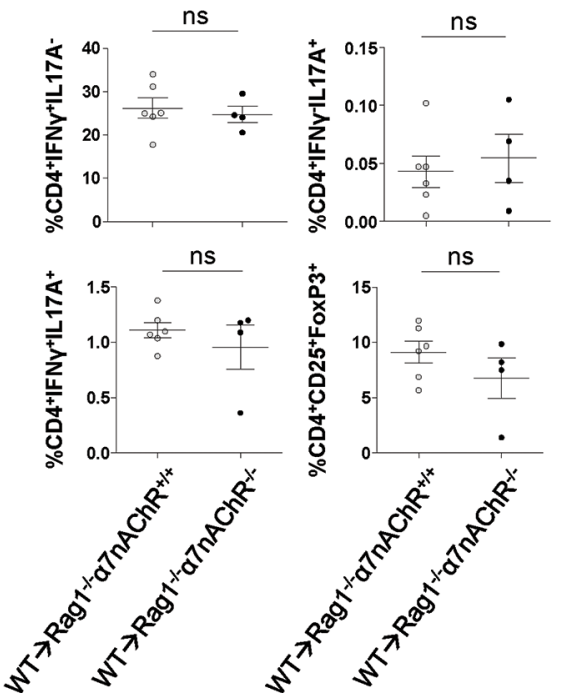

Figure 7. Severity of T cell transfer induced colitis is independent of $\alpha$ 7nAChR signaling. Naïve T cells isolated by cell sorting from WT mice, were adoptively transferred i.p. in

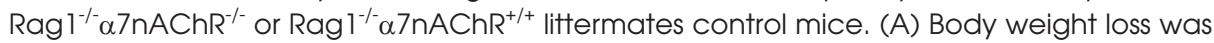
assessed twice per week. Statistical significance was determined using a multivariate linear model after a post-hoc Bonferroni-Holm correction for body weight changes and stool consistency score (ns, not significant). (B) Five weeks after adoptive transfer, mice were sacrificed and disease severity was additionally assessed by means of colon length and spleen weight. (C) Inflammatory gene expression was measured by q-PCR in the colon. Gene expression levels are shown relative to rp/32 mRNA expression. (D) Frequencies and numbers of Thelper cell and Treg subsets were assessed in the colon by flow cytometry. Dots represent individual mice. Data expressed as mean \pm SEM are representative of 3 independent experiments. Statistical significance was determined with the unpaired Student t-test for colon length, spleen weight, gene expression level and flow cytometry analysis (ns, not significant).

in MLNs, confirming that impairment of vagal tone might affect Treg expansion. Our findings are in line with a previous study showing that VGX resulted in increased basal levels of NF- $\kappa \mathrm{B}$, leading to a more severe colitis after colonic exposure to DSS (7). Similarly, Morishita et al. recently showed that electrical stimulation of the vagus nerve increased the
Treg-to-Th17 ratio in MLNs in a rat model of trauma/hemorrhagic shock (27).

Although our results support the hypothesis that the vagus nerve plays a role in the maintenance of intestinal immune homeostasis, VGX did not influence severity of $T$ cell transfer model of chronic colitis. Indeed, disease activity and body weight loss were similar in both VGX Rag1/-- and sham Rag1/-. This observation is in line with a previous report by van der Kleij $\mathrm{H}$ et al., showing that VGX had no effect in chronic model of colitis (28). Interestingly, these data are in contrast with the worsening effect of VGX in DSS colitis models. It should be emphasized that the mechanisms involved in these two models differ substantially. DSS treatment typically causes disruption of the mucosal barrier triggering a complex immune response against intraluminal antigens, a process mainly mediated by activation of lamina propria M $\varphi s$ (29). The latter represent both the target population of the cholinergic immunomodulation $(6,30)$ and crucial player in Treg proliferation (23), most likely explaining the increased severity of DSS colitis following VGX. In contrast, $\mathrm{T}$ cell transfer model of chronic colitis is associated with uncontrolled proliferation and activation of $\mathrm{T}$ cells transferred into an immune-deficient recipient, with accumulation of effector Th1 and Th17 cells in the intestine (31). Second, and most importantly, previous experiments elegantly showed that increased susceptibility to develop colitis following VGX only lasts 2-3 wks due to a compensatory increase in cholinergic tone generated by the enteric nervous system (26). As the onset of colitis only becomes obvious $2-3$ wks following $\mathrm{T}$ cell transfer, the effect of VGX on the development of colitis will be minimal or even absent. Future experiments evaluating the effect of repetitive VNS on $\mathrm{T}$ cell transfer colitis may be better suited to evaluate the potential immunomodulatory effect of cholinergic input, but this approach is currently technically not feasible.

Considering the key role of $\alpha 7 \mathrm{nAChR}$ in the cholinergic anti-inflammatory pathway, we performed investigations on $\alpha 7 \mathrm{nAChR}^{-/-}$mice to determine the importance of this receptor in the intestinal mucosal immune compartment during both oral tolerance induction and colitis. On the contrary, to data reported in postoperative ileus and sepsis, $\alpha 7 \mathrm{nAChR}$ was not involved 
in the development of oral tolerance. In line, $\alpha 7 \mathrm{nAChR}{ }^{-/-}$mice developed similar degree of DSS colitis as in littermate control $\alpha 7 \mathrm{nAChR}^{+/+}$mice, suggesting that $\alpha 7 \mathrm{nAChR}$ do not alter the immune response during DSS colitis. Similarly, no differences in colitis severity were observed between $\operatorname{Rag} 1^{-/-}$ mice transferred with $\alpha 7 \mathrm{nAChR}^{+/+}$or $\alpha 7 \mathrm{nAChR}^{-/-}$naïve $\mathrm{T}$ helper cells indicating that $\alpha 7 \mathrm{nAChR}$ on $\mathrm{T}$ cells are not crucial for maintaining intestinal $\mathrm{T}$ cell homeostasis. In line, although previous studies have described expression of $\alpha 7 n A C h R$ on T cells (32-35), we could not confirm $\alpha 7 \mathrm{nAChR}$ expression on naïve or polarized $T$ cells (data not shown). Finally, $\alpha 7 \mathrm{nAChR}{ }^{+/+} \mathrm{Rag}^{-/-}$and $\alpha 7 \mathrm{nAChR}^{-/-} \mathrm{Rag}^{1 /-}$ recipient mice adoptively transferred with WT naïve T cells developed comparable colitis, indicating that $\alpha 7 n A C h R$ does not have a crucial role during development of $\mathrm{T}$ cell transfer-induced chronic colitis. So far, contradictory results concerning the role of $\alpha 7 \mathrm{nAChR}$ in colitis have been reported. While $\alpha 7 \mathrm{nAChR}{ }^{-/-}$mice showed higher severity of depression-induced colitis (11), treatment with specific $\alpha 7 \mathrm{nAChR}$ agonists (AR-R17779 and GSK1345038A) reduced inflammation such as NF- $\mathrm{BB}$ activity and cytokines but aggravated clinical parameters of DSS and TNBS colitis (36). In addition, $\alpha 7 \mathrm{nAChR}$ agonists reduced IL-12p70 and IL-23 production by CD11c ${ }^{+}$DCs and their ability to prime T cells in vitro $(12,13)$. Overall, our data fail to support a fundamental role for $\alpha 7 \mathrm{nAChR}$ in modulating intestinal mucosal immune response. Although we showed a significant immunomodulatory role for the vagus nerve in both oral tolerance and colitis, our data suggest the involvement of other neuro-receptors. In line, activation of the M1 muscarinic acetylcholine receptor or treatment with acetyl-cholinesterase inhibitors reduced the immune response in colitis models $(12,13)$, indicating that not only nicotinic receptors agonist can modulate colitis. In addition, a possible redundancy between the different nicotinic receptors could explain our results. $\beta 2$ nicotinic receptors $(\beta 2 \mathrm{nAChR})$ have indeed been detected on M $\varphi s$, while a $\beta 2 \mathrm{nAChR}$ agonist inhibited ATP-induced M $\varphi s$ activation in the stomach (37). Similarly, $\alpha 4 \beta 2$ nicotinic receptor activation inhibits isolated intestinal and peritoneal M $\varphi s$ (38) and $\alpha 5$ nicotinic receptor knockout mice showed a significant worsening of disease compared with WT mice during colitis (39).

\section{CONCLUSION}

Overall, our results showed that VGX impaired oral tolerance induction, decreased proliferation of Tregs and exacerbated DSS colitis, suggesting the vagus nerve is an important player in the intestinal immune homeostasis. Our findings have translational implications and indeed suggest that activation of the CAIP might be useful as a therapeutic tool to prevent diseases associated with intestinal inflammation and impaired oral tolerance. However, the underlying mechanisms such as the receptor involved need to be further investigated.

\section{ACKNOWLEDGMENTS}

The authors would like to thank K. Lambaerts, R. De Keyser, Inne Croux and Iris Appeltans (Translational Research Center in Gastrointestinal Disorders, KU Leuven) for their technical assistance, Petra Vandervoort (Molecular and Vascular Biology Research Unit, KU Leuven) for assistance with cell sorting and Isabel Vogel Geb Zinsser for her skilled technical help with intravenous injection.

\section{DISCLOSURE}

This work was supported by funding from the European Research Council (ERC) Advanced Grant (ERC-2013Adg): 340101 Cholstim. This work was supported by grants from the Research Foundation - Flanders (FWO) (Odysseus and Hercules program to GEB), by a FWO PhD fellowship (to MDG) and by a FWO postdoctoral research fellowship (to GM and PJG).

\section{REFERENCES}

1. Sternberg EM (2006) Neural regulation of innate immunity: a coordinated nonspecific host response to pathogens. Nat Rev Immunol 6(4):318-28.

2. Borovikova LV, Ivanova $S$, Zhang $M$, Yang $H$, Botchkina GI, Watkins LR, et al. (2000) Vagus nerve stimulation attenuates the systemic inflammatory response to endotoxin. Nature 405(6785):458-62.

3. Wang H, Yu M, Ochani M, Amella CA, Tanovic M, Susarla S, et al. (2003) Nicotinic acetylcholine receptor alpha7 subunit is an essential regulator of inflammation. Nature 421(6921):384-8.

4. Tracey KJ (2002) The inflammatory reflex. Nature 420(6917):853-9.

5. de Jonge WJ, Van Der Zanden EP, The FO, Bijlsma MF, van Westerloo DJ, Bennink RJ, et al. (2005) Stimulation of the vagus nerve attenuates macrophage activation by activating the Jak2-STAT3 signaling pathway. Nat Immunol 6(8):844-51.

6. Matteoli G, Gomez-Pinilla PJ, Nemethova A, Di GM, Cailotto C, van Bree SH, et al. (2014) A distinct vagal anti-inflammatory pathway modulates intestinal muscularis resident macrophages independent of the spleen. Gut 63(6):938-48.

7. O'Mahony C, van der Kleij H, Bienenstock J, Shanahan F, O'Mahony L (2009) Loss of vagal anti-inflammatory effect: in vivo visualization and adoptive transfer. Am. J. Physiol. Regul Integr Comp Physiol 297(4):R1118-R1126.

8. Ghia JE, Blennerhassett P, Kumar-Ondiveeran H, Verdu EF, Collins SM (2006) The vagus nerve: a tonic inhibitory influence associated with inflammatory bowel disease in a murine model. Gastroenterology 131(4):1122-30.

9. Ghia JE, Blennerhassett P, El-Sharkawy RT, Collins SM (2007) The protective effect of the vagus nerve in a murine model of chronic relapsing colitis. Am. J. Physiol. Gastrointest Liver Physiol 293(4):G711-G718.

10. Ghia JE, Blennerhassett P, Collins SM (2008) Impaired parasympathetic function increases susceptibility to inflammatory bowel disease in a mouse model of depression. J. Clin. Invest. 118(6):2209-18.

11. Ghia JE, Blennerhassett P, Deng Y, Verdu EF, Khan WI, Collins SM (2009) Reactivation of inflammatory bowel disease in a mouse model of depression. Gastroenterology 136(7):2280-8.

12. Ji H, Rabbi MF, Labis B, Pavlov VA, Tracey KJ, Ghia JE (2014) Central cholinergic activation of a vagus nerve-to-spleen circuit alleviates experimental colitis. Mucosal Immunol 7(2):335-47.

13. Munyaka P, Rabbi MF, Pavlov VA, Tracey KJ, Khafipour E, Ghia JE (2014) Central muscarinic cholinergic activation alters interaction between splenic dendritic cell and CD4+. PLoS One 9(10):e109272.

14. Pabst O, Mowat AM (2012) Oral tolerance to food protein. Mucosal Immunol 5(3):232-9. 
15. Hadis U, Wahl B, Schulz O, Hardtke-Wolenski M, Schippers A, Wagner N, et al. (2011) Intestinal tolerance requires gut homing and expansion of FoxP3+ regulatory $\mathrm{T}$ cells in the lamina propria. Immunity 34(2):237-46.

16. Haribhai D, Lin W, Edwards B, Ziegelbauer J, Salzman NH, Carlson MR, et al. (2009) A central role for induced regulatory $\mathrm{T}$ cells in tolerance induction in experimental colitis. J. Immunol. 182(6):3461-8.

17. Tanner SM, Staley EM, Lorenz RG (2013) Altered generation of induced regulatory $\mathrm{T}$ cells in the FVB.mdr1a-/- mouse model of colitis. Mucosal Immunol 6(2):309-23.

18. Bencherif M, Lippiello PM, Lucas R, Marrero MB (2011) Alpha7 nicotinic receptors as novel therapeutic targets for inflammation-based diseases. Cell Mol Life Sci 68(6):931-49.

19. Mombaerts P, Iacomini J, Johnson RS, Herrup K, Tonegawa S, Papaioannou VE (1992) RAG-1deficient mice have no mature $\mathrm{B}$ and $\mathrm{T}$ lymphocytes. Cell 68(5):869-77.

20. Wirtz S, Neufert C, Weigmann B, Neurath MF (2007) Chemically induced mouse models of intestinal inflammation. Nat Protoc 2(3):541-6.

21. Read S, Malmstrom V, Powrie F (2000) Cytotoxic T lymphocyte-associated antigen 4 plays an essential role in the function of CD25(+)CD4(+) regulatory cells that control intestinal inflammation. J. Exp. Med. 192(2):295-302.

22. Sun CM, Hall JA, Blank RB, Bouladoux N, Oukka M, Mora JR, et al. (2007) Small intestine lamina propria dendritic cells promote de novo generation of Foxp3 $\mathrm{T}$ reg cells via retinoic acid. J. Exp. Med. 204(8):1775-85.

23. Hadis U, Wahl B, Schulz O, Hardtke-Wolenski M, Schippers A, Wagner N, et al. (2011) Intestinal tolerance requires gut homing and expansion of FoxP3+ regulatory $\mathrm{T}$ cells in the lamina propria. Immunity 34(2):237-46.

24. Weiner HL, da Cunha AP, Quintana F, Wu H (2011) Oral tolerance. Immunol. Rev. 241(1):241-59.

25. Ortega-Gomez A, Perretti M, Soehnlein O (2013) Resolution of inflammation: an integrated view. EMBO Mol. Med. 5(5):661-74.

26. Ghia JE, Blennerhassett P, Collins SM (2007) Vagus nerve integrity and experimental colitis. Am. J. Physiol. Gastrointest Liver Physiol 293(3):G560-G567.

27. Morishita K, Coimbra R, Langness S, Eliceiri BP, Costantini TW (2015) Neuroenteric axis modulates the balance of regulatory $\mathrm{T}$ cells and T-helper 17 cells in the mesenteric lymph node following trauma/hemorrhagic shock. Am. J. Physiol. Gastrointest Liver Physiol 309(3):G202-G208.

28. van der Kleij H, O'Mahony C, Shanahan F, O'Mahony L, Bienenstock J (2008) Protective effects of Lactobacillus rhamnosus [corrected] and Bifidobacterium infantis in murine models for colitis do not involve the vagus nerve. Am. J. Physiol. Regul Integr Comp Physiol 295(4):R1131-R1137.

29. Chassaing B, Aitken JD, Malleshappa M, Vijay-Kumar M (2014) Dextran sulfate sodium
(DSS)-induced colitis in mice. Curr Protoc Immunol 104:Unit.

30. de Jonge WJ, Van Der Zanden EP, The FO, Bijlsma MF, van Westerloo DJ, Bennink RJ, et al. (2005) Stimulation of the vagus nerve attenuates macrophage activation by activating the Jak2-STAT3 signaling pathway. Nat Immunol 6(8):844-51.

31. Ostanin DV, Bao J, Koboziev I, Gray L, Robinson-Jackson SA, Kosloski-Davidson M, et al. (2009) T cell transfer model of chronic colitis: concepts, considerations, and tricks of the trade. Am. J. Physiol. Gastrointest Liver Physiol 296(2):G135-G146.

32. Liu Z, Han B, Li P, Wang Z, Fan Q (2014) Activation of alpha7nAChR by nicotine reduced the Th17 response in CD4(+)T lymphocytes. Immunol Invest 43(7):667-74.

33. de Jonge WJ, Ulloa L (2007) The alpha7 nicotinic acetylcholine receptor as a pharmacological target for inflammation. Br. J. Pharmacol. 151(7):915-29.

34. Van Der Zanden EP, Hilbers FW, Verseijden C, Van den Wijngaard RM, Skynner M, Lee K, et al. (2012) Nicotinic acetylcholine receptor expression and susceptibility to cholinergic immunomodulation in human monocytes of smoking individuals. Neuroimmunomodulation 19(4):255-65.

35. Razani-Boroujerdi S, Boyd RT, Davila-Garcia MI, Nandi JS, Mishra NC, Singh SP, et al. (2007) $\mathrm{T}$ cells express alpha7-nicotinic acetylcholine receptor subunits that require a functional TCR and leukocyte-specific protein tyrosine kinase for nicotine-induced Ca2+ response. J. Immunol. 179(5):2889-98.

36. Snoek SA, Verstege MI, Van Der Zanden EP, Deeks N, Bulmer DC, Skynner M, et al. (2010) Selective alpha7 nicotinic acetylcholine receptor agonists worsen disease in experimental colitis. Br. J. Pharmacol. 160(2):322-33.

37. Nemethova A, Michel K, Gomez-Pinilla PJ, Boeckxstaens GE, Schemann M (2013) Nicotine attenuates activation of tissue resident macrophages in the mouse stomach through the beta2 nicotinic acetylcholine receptor. PLoS One 8(11):e79264.

38. Van Der Zanden EP, Snoek SA, Heinsbroek SE, Stanisor OI, Verseijden C, Boeckxstaens GE, et al. (2009) Vagus nerve activity augments intestinal macrophage phagocytosis via nicotinic acetylcholine receptor alpha4beta2. Gastroenterology 137(3):1029-39, 1039.

39. Orr-Urtreger A, Kedmi M, Rosner S, Karmeli F, Rachmilewitz D (2005) Increased severity of experimental colitis in alpha 5 nicotinic acetylcholine receptor subunit-deficient mice. Neuroreport. 16(10):1123-7.

Cite this article as: Di Giovangiulio M, et al. (2016) Vagotomy affects the development of oral tolerance and increases susceptibility to develop colitis independently of $\alpha-7$ nicotinic receptor. Mol. Med. 22:464-76. 